\title{
La clase inversa y el aprendizaje servicio: fusión de dos metodologías convergentes para el aprendizaje de lengua extranjera
}

\section{Daniela Gil-Salom ${ }^{a}$ Ángeles Lence ${ }^{b}$}

Departamento de Lingüística Aplicada, Universitat Politècnica de València, ${ }^{a}$ dagil@idm.upv.es, b $\underline{\text { malence@,idm.upv.es. }}$

\begin{abstract}
This work presents the result of fusing two pedagogical active methodologies,: flipped classroom and service learning, in the teaching of two transversal subjects of foreign languages: German and French. Therefore, the implementation of the flipped classroom by carrying out a service-learning project is shown and the way they complement each other is highlighted with practical examples of two different levels of the ERFL, German A1 and French B1. With the results, it can be seen, that the two methodologies not only match perfectly, but the flipped classroom is being implemented by developing a service-learning project. With the aim of designing a good articulated project with social commitment, students learn the content in a gratifying way, reflect on their direct or indirect implication in the community and develop skills for their future profession.
\end{abstract}

Keywords: flipped classroom, service learning, transversal skills, German as a foreign language, French as a foreign language.

\begin{abstract}
Resumen
Este trabajo presenta el resultado de fusionar dos metodologías pedagógicas activas, como son la clase inversa (CI) y el aprendizaje-servicio (ApS), en la docencia de dos asignaturas transversales de lengua extranjera: alemán y francés. Para ello, mostraremos la implementación de la clase inversa llevando a cabo un proyecto ApS y destacaremos cómo se complementan, con ejemplos prácticos en dos niveles distintos del MCREL, Alemán A1 y Francés B1. Con el resultado, veremos que se trata de dos metodologías que no solo casan perfectamente, sino que la CI se implementa al llevar a cabo un proyecto ApS: con el objetivo de diseñar un proyecto bien articulado con compromiso social, el alumnado aprende la materia de forma gratificante, reflexiona sobre su implicación directa o indirecta en la comunidad y desarrolla competencias para su futura profesión.
\end{abstract}

Palabras clave: clase inversa, aprendizaje-servicio, competencias transversales, alemán como lengua extranjera, francés como lengua extranjera. 


\section{Introducción}

En los últimos cursos, la UPV ha apostado por dos metodologías innovadoras para la práctica docente en contexto politécnico: la clase inversa (CI) y el aprendizaje-servicio (ApS). La primera arrancó con la experiencia piloto en el curso 2014/15 y la segunda en el curso 2016/17, aunque ambas estaban ya presentes sin el reconocimiento académico.

La CI es un modelo pedagógico centrado en el alumno que se fundamenta en la utilización de tecnologías digitales al servicio de la enseñanza-aprendizaje (Barreras, MA, 2016: 176). Al mismo tiempo, posibilita que el aula se convierta en un espacio de aprendizaje creativo y reflexivo. De este modo, el docente tiene una visión global del aprendizaje ya que diseña técnicas o actividades didácticas que le permiten desarrollar diferentes enfoques metodológicos.

El ApS es también un modelo pedagógico centrado en el alumno, especialmente en su compromiso social, que combina el aprendizaje a través de la experiencia y la acción al servicio de la comunidad: el alumnado aprende dando un servicio a la sociedad. Este aprendizaje se desarrolla en el aula que se convierte, al igual que en la CI, en un espacio participativo, creativo y reflexivo, donde el docente incentiva la autonomía del alumno (García, 2016:215) y el trabajo en equipo con el objetivo de realizar un proyecto bien articulado.

En este trabajo pretendemos exponer cómo ambas metodologías son perfectamente aplicables puesto que se complementan y se apoyan la una en la otra con idénticos objetivos en una asignatura.

\section{Objetivos}

Los objetivos de este trabajo son, por un lado, ejemplificar la implementación de la metodología de la clase inversa llevando a cabo un proyecto ApS, y por otro, destacar cómo se complementan ambos enfoques metodológicos

Para ello, vamos a desarrollar dos ejemplos prácticos en dos asignaturas de lengua extranjera: alemán y francés en dos niveles distintos del Marco Común de Referencia Europeo de las Lenguas (MCREL) ${ }^{1}$, A1 y B1 respectivamente.

\section{Desarrollo de la innovación}

A continuación se presentan las dos experiencias que se han llevado a cabo durante el pasado curso 2016/17 en Alemán A1 y Francés B1.

\subsection{La asignatura Alemán 1}

3.1.1. Contexto académico: se trata de una asignatura optativa transversal de la UPV, formada por siete grupos de estudiantes pertenecientes a diez titulaciones de ocho ERT distintas, con una matrícula de 170 personas y una carga docente de 4,5 / 6 créditos según titulación. El proyecto ApS se llevó a cabo en un grupo ubicado en la ETSINF y

\footnotetext{
${ }^{1}$ MCERL http://cvc.cervantes.es/ensenanza/biblioteca ele/marco/default.htm
} 
constaba de 20 estudiantes de las titulaciones Grado en Ingeniería Informática y Grado en Ingeniería de Organización Industrial.

3.1.2. Proyecto ApS: el proyecto diseñado para esta experiencia se titula "PROYECTO graTICs": aprendizaje del alemán apoyado en herramientas TIC gratuitas. La idea en la que se sustenta este proyecto es fomentar el aprendizaje de la lengua alemana en la enseñanza pública no universitaria, dada su importancia en el terreno académico y profesional contemporáneo. Al mismo tiempo, la puesta en práctica de la experiencia desarrolla las competencias transversales y específicas de la asignatura.

3.1.3. Objetivos del proyecto ApS: con este proyecto se pretende llamar la atención sobre la falta de oferta de lengua alemana en los centros públicos. Es una carencia formativa que sufre la mayoría del alumnado de la enseñanza pública, tanto de secundaria y bachiller, como de módulos formativos.

3.1.4. Objetivos académicos: reforzar y asentar conocimientos como la pronunciación, el vocabulario, la morfología y la sintaxis de la lengua alemana, así como desarrollar todas las competencias transversales, especialmente: Comprensión e integración (CT01), Aplicación y pensamiento práctico (CT02), Diseño y proyecto (CT05), Trabajo en equipo y liderazgo (CT06), Comunicación efectiva (CT08), Conocimiento de problemas contemporáneos (CT10) o Aprendizaje permanente (CT11). Pero sobre todo, es importante crear una oportunidad para iniciar proyectos interdisciplinares, al trabajar de forma creativa estudiantes de distintas titulaciones en objetivos comunes.

3.1.5. Metodología: el trabajo dedicado a este proyecto se desarrolla tanto en sesiones presenciales como no presenciales y es de carácter individual o grupal, según la actividad programada.

Las fases que componen este proyecto ApS son las siguientes:

1. Lectura previa de documentos sobre el nuevo enfoque metodológico y ejemplos de proyectos similares, así como de contenidos teóricos de la materia de la asignatura, todo ello ubicado en Recursos de PoliformaT. Esta preparación previa a las clases presenciales responde a los fundamentos de la metodología propia de la CI.

2. Formación de grupos de trabajo: el alumnado se distribuye en grupos de tres/cuatro personas para definir el pre-diseño de los distintos sub-proyectos de forma presencial. El trabajo colaborativo es propio de la metodología ApS y es igualmente protagonista en las actividades presenciales asociadas a la clase inversa.

3. Búsqueda de fuentes y material. Trabajo presencial y no presencial. En esta fase se combina la investigación individual del alumnado fuera del aula (proceso que comparten ambas metodologías) con la puesta en común (fase de comunicación entre pares para la toma de decisiones) con el resto del grupo, para decidir aquellas fuentes y herramientas a utilizar. Aquí confluyen CI y ApS de una manera significativa para desarrollar estas competencias transversales. 
4. Diseño final de los sub-proyectos en sesiones no presenciales. Dos grupos se dedican a la aplicación de diseño propio y cuatro grupos a las aplicaciones y herramientas gratuitas en línea.

5. Desarrollo de la acción durante dos sesiones presenciales. Desplazamiento a tres aulas de Ciclos Formativos de Informática del IES Serpis de Valencia. Puesta en práctica de los conocimientos adquiridos en sesiones de trabajo presenciales y sesiones no presenciales en un contexto real.

6. Resultados del proyecto: según la reflexión de los estudiantes, en general el proyecto favorece el aprendizaje y refuerzo de los contenidos de la asignatura. De forma voluntaria, cuatro grupos realizaron un vídeo para darle visibilidad al proyecto mediante su difusión en páginas web de instituciones educativas, tales como el mismo IES Serpis y la UPV.

7. Evaluación del proyecto: por un lado, los destinatarios del proyecto evalúan a sus "formadores" mediante un cuestionario y, por otro, los estudiantes que realizan el servicio evalúan todo el proceso a través de un texto reflexionando sobre el proyecto global, el del grupo y sobre la propia actuación a nivel individual.

3.1.6. Evaluación: para la evaluación académica se diferencian igualmente dos niveles: valoración individual, basada en un texto de reflexión y valoración del trabajo en grupo según el grado de implicación en el proyecto, cada uno con un peso de 0,5 puntos de la nota final.

3.1.7. Resultados: en lo relativo a la competencia lingüística, se observa un mejor aprendizaje de la lengua alemana según la percepción de los estudiantes, tal como se observa en los vídeos presentados como trabajo final del proyecto; un ejemplo de ello se muestra en la página web del Centro de Cooperación al Desarrollo (CCD) de la UPV: https://youtu.be/HgJOOwW4yng. El diseño de materiales llevado a cabo por los propios estudiantes desarrolla así el proceso metacognitivo descrito por Flavell (1985). Del mismo modo, el propio diseño se ve enriquecido por la interdisciplinariedad del perfil académico del alumnado. En cuanto a la competencia cívica, supone una oportunidad para concienciar al alumnado de su responsabilidad individual ante la sociedad.

\subsection{La asignatura Francés B1}

3.2.1. Contexto académico: se trata de una asignatura optativa transversal de la UPV, que consta de dos grupos de estudiantes pertenecientes a veinte titulaciones de diez ERT distintas, con una matrícula de 106 personas y una carga docente de 4,5 / 6 créditos según titulación. El proyecto ApS se llevó a cabo en el grupo ubicado en la ETSIE y constaba de 10 estudiantes de distintos Grados (Grado en Ingeniería Industrial, Grado en Biotecnología y Grado en Administración y Dirección de Empresas).

3.2.2. Proyecto ApS: "Posters para el ApS: difusión bilingüe (castellano/francés) de proyectos de Origines". El proyecto se basa en la difusión de los proyectos de educación e inserción laboral que la asociación Origines lleva a cabo en dos islas senegalesas, Fadiouth y Ehidj. Simultáneamente, la puesta en práctica de la experiencia desarrolla las competencias transversales y específicas de la asignatura (Lence, 2014).

(cc)) EY-NC-ND 2017, Universitat Politècnica de València 
3.2.3. Objetivos académicos: adquirir mejor competencia lingüística poniendo en práctica la comunicación efectiva (CT-8), comprender los objetivos de la tarea e integrarlos sin esfuerzo en su formación (CT-01); aprender a desarrollar un pensamiento crítico (CT2); aprender a ser creativo en una actividad innovadora en la UPV (CT-04) al seleccionar y manipular imágenes, buscar la originalidad en la presentación; aprender a diseñar y presentar un proyecto (CT-05), fundamental para su PFG; aprender a trabajar en equipo y liderar (CT.06), pues hay que distribuir el trabajo, negociar, decidir y coordinar; aprender a ser responsable, a tener un comportamiento ético y de respeto hacia los demás (CT-07); aprender a reconocer los problemas contemporáneos importantes (CT-9) como la brecha educativa; experimentar una inmersión intercultural, un aprendizaje permanente (CT-10) que le motive a saber más sobre otros contextos; aprender a gestionar y planificar el tiempo (CT-11) para que los resultados sean acordes al tiempo establecido sin someterse a estrés alguno; por último, aprender a ser consciente de lo importantes que son sus estudios específicos para transformar la sociedad (Lence, 2016:457).

3.2.4. Objetivos del proyecto ApS: para la difusión de los proyectos de Origines, se trata de involucrar al alumnado en la producción de material que dé visibilidad a los mismos para su presentación en contextos académicos como es el caso del congreso ApSU17 que se celebrará en octubre próximo. A tal fin y con la participación activa del alumnado en jornadas o congresos de cooperación al desarrollo o de aprendizaje de lenguas, se pretende igualmente producir conocimiento a través del debate entre ponentes y de las intervenciones del público en general.

Al mismo tiempo, buscamos promover la concienciación en la sociedad dando a conocer la realidad de otro país y las líneas de acción que lleva a cabo una ONG como Origines para mejorar la situación de niños, niñas y jóvenes en riesgo de abandono escolar o de exclusión social.

3.2.5. Metodología: partiendo del enfoque de las metodologías activas, la puesta en marcha del proyecto se estructura del siguiente modo: de los 6 créditos de los que consta la asignatura, hemos dedicado a ApS 0,5 créditos TA/PA, que corresponden a dos sesiones presenciales y 2 créditos PL, correspondientes a seis sesiones de prácticas.

Las fases que componen este proyecto ApS son las siguientes:

1. Presentación del profesor de documentos sobre el nuevo enfoque metodológico y ejemplos: definición, descripción y aplicación del ApS, con el material alojado en Recursos de PoliformaT. (TA/PA1)

2. Presentación de Moussa Sarr, coordinador de Origines, sobre Senegal y los entornos en los que se llevan a cabo los proyectos de educación y de inserción laboral (islas Fadiouth, Ehidj ...). (TA/PA2)

3. Formación del grupo de trabajo y distribución por binomios: pre-diseño de los distintos apartados. (PL1)

4. Búsqueda de fuentes y material para elaborar el póster. (PL2)

5. Puesta en común y diseño del póster. (PL3) 
6. Desarrollo de la acción: presentación del póster en contexto francófono. (PL4)

7. Resultados del proyecto. (PL5)

8. Evaluación del proyecto que incluye reflexión. (PL6)

La presentación real del póster en castellano tendrá lugar en el VIII Congreso Nacional e Internacional ApSU en Sevilla, del 13 al 14 de octubre de 2017. Se trata además de un proyecto financiado por GLOCAL2017.

3.2.6. Evaluación: para la evaluación académica se establecen dos niveles: una valoración individual, a partir de un texto de reflexión y una valoración del trabajo en equipo que valora la participación en el proyecto, con un peso de 1 punto de la nota final.

\subsubsection{Resultados}

Los resultados generales del proyecto reflejan la adquisición de la lengua francesa desarrollando a la vez las competencias transversales. Gracias al proyecto, se materializa un servicio a la sociedad como es la difusión de los proyectos de una ONG. Con ello, se contribuye a la mayor visibilidad de actividades relacionadas con el ApS en la UPV. Además, vemos aumentada la motivación del alumnado por la tarea gratificante de procurar un mundo más justo (Lence, 2015:61).

\section{Resultados conjuntos}

Desde el punto vista del alumnado, la aplicación de la CI y del ApS supone una vía innovadora de aprendizaje e investigación a la vez que permite despertar o reforzar los valores cívicos y de concienciación sobre la realidad más allá de las aulas. La práctica significativa del saber facilita el acercamiento a problemas contemporáneos y a la reflexión, no solo sobre el aprendizaje, sino también sobre uno mismo y sobre los demás, desarrollando el espíritu crítico como nos apunta Deeley (2016:164) dándole así un sentido más amplio a ese aprendizaje.

Desde la perspectiva del docente, la aplicación de técnicas y enfoques nuevos, aunque al principio suponga un esfuerzo añadido (Gil-Salom, 2015), proporciona una gran satisfacción profesional, difícil de medir cuantitativamente, ya que depende, como en el caso del alumnado, del grado de implicación.

Desde la propia experiencia, sentimos el impulso de querer saber más sobre ambos enfoques, haciendo hincapié en el ApS puesto que se trata de una metodología en la que aún existen lagunas, especialmente en lo relativo a la evaluación y que, por otro lado, es un enfoque que aún no siendo nuevo, sí lo es en la UPV (Macías-Mendoza, MartínezUsarralde \& Gil-Salom, 2016; Macías-Mendoza \& Gil-Salom, 2017).

\section{Conclusiones}

Todos estamos de acuerdo en la importancia del aprendizaje activo, reflexivo, autónomo y responsable. Hemos visto que la CI y el ApS son dos metodologías que pueden perfectamente fusionarse para el aprendizaje de lengua extranjera, tanto si se trata de un

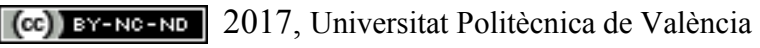


nivel de iniciación (A1) como de uno intermedio (B1), pues ambas implementan el aprendizaje de las competencias lingüísticas y transversales.

En el caso de la lengua alemana, la asignatura de Alemán A1 ya contaba con la experiencia de cursos anteriores en CI al formar parte del proyecto del mismo nombre iniciado por la UPV en el curso 2014/15, pero ha sido con esta experiencia con la que se inicia en la metodología ApS.

En el caso de la lengua francesa, la asignatura de Francés B1, reconocida institucionalmente como asignatura asociada al ApS, la CI se ha aplicado hasta ahora solo de forma intuitiva, al no haberse solicitado en tiempo y forma su inclusión como CI. Sin embargo, al comprobar que ambas metodologías se fusionan perfectamente, hemos solicitado su reconocimiento para el próximo curso en la asignatura de Francés 2 (B2) habiendo sido aceptada.

Tal como hemos indicado en los resultados conjuntos, a partir de estas experiencias, uno de nuestros objetivos es profundizar, tanto en el enfoque metodológico de la CI, como en la metodología ApS para optimizar la programación docente en asignaturas de lengua extranjera.

Por último, confiamos en que este trabajo pueda contribuir a la consolidación de las competencias transversales definidas por la UPV, especialmente de la que se refiere a la "Responsabilidad ética, medioambiental y profesional" para que esta universidad, referente en desarrollo tecnológico, destaque igualmente "por sus compromisos en materia de responsabilidad social” respondiendo a su Plan Estratégico 2020.

\section{Referencias}

BARRERAS GÓMEZ, M.A. (2016) Experiencia de la clase inversa en didáctica de las lenguas extranjeras, Educatio Siglo XXI, vol. 34, issue 1, p. 173-196, http://dx.doi.org/10.6018/j/253281

DEELEY, S.J. (2016) El aprendizaje-servicio en educación superior. Teoría, práctica y perspectiva crítica. Madrid: Narcea.

FlaVell, J.H. 1985. Cognitive development. Englewood Cliffs. NJ: Prentice Hall.

GARCÍA GómEZ, A. (2016) Aprendizaje inverso y motivación en el aula universitaria, Pulso. Revista de Educación, 39, 199-218.

GIL-SALOM, D. (2015) “Alemán para Grado en Ingeniería Informática aplicando flipped teaching”, en Skorczynska Sznajder, H., Carrió Pastor, M. L., Saz Rubio, M. M., Tamarit Vallés, I. T. Estudios de lingüística aplicada I Valencia: Universitat Politècnica de València. http://hdl.handle.net/10251/53329.

LENCE GUILABERT, A. (2016) “Aprendizaje y servicio en asignaturas de francés: difusión del alumnado del proyecto educativo de Origines en Senegal", Actas VII Congreso Nacional y II Internacional de Aprendizaje-Servicio Universitario (APS U7), p 451-459, Universidade de Santiago de Compostela. http: //dx.doi.org/10.15304/978841653397. 
LENCE GUILABERT, A. (2015) Formation et évaluation en compétences interculturelles pour ingénieurs, Cuadernos de Filología Francesa, $\mathrm{n}^{\circ}$ 26, Universidad de Extremadura, p 45-63. http://hdl.handle.net/10251/78992

LenCE Guilabert, A. (2014) “Cooperación para el desarrollo en educación: Origines en Francés II y competencias transversales" Actas II Jornadas de Innovación Docente en la ETSIT (JIDTEL'2014). En homenaje a Elvira Bonet, Editorial Universitat Politècnica de València.

MacíAs-MendozA, D., MARTíneZ-Usarralde, M.J. y GIL-SAlOM, D. (2016) "La formación en competencias transversales y el Aprendizaje Servicio (ApS): feliz maridaje en la Universitat Politècnica de València" en Fernández Prada, MÁ.; Botti Navarro, VJ. (2016). In-Red 2016. II Congreso nacional de innovación educativa y docencia en red. Editorial Universitat Politècnica de València. http://hdl.handle.net/10251/70954.

MACÍAS-MENDOZA, D. Y GIL-SALOM, D. (2017). Aprender y enseñar lenguas. (en prensa)

MCERL http://cvc.cervantes.es/ensenanza/biblioteca_ele/marco/default.htm

Plan Estratégico de la Universitat Politècnica de València 2020 https://www.upv.es/organizacion/la-institucion/documentos/Plan_Estrategico_UPV_20152020 Resumen_ejecutivo.pdf 